\title{
Situation monitoring of urban areas using social media data streams
}

\author{
Andreas Weiler*, Michael Grossniklaus, Marc H. Scholl \\ Department of Computer and Information Science, University of Konstanz, Germany
}

\section{A R T I C L E I N F O}

Keywords:

Urban situation visualization

Event and topic detection and tracking

Twitter social data stream

\begin{abstract}
A B S T R A C T
The continuous growth of social networks and the active use of social media services result in massive amounts of user generated data. Our goal is to leverage social media users as "social sensors" in order to increase the situational awareness within and about urban areas. In addition to the well known challenges of event and topic detection and tracking, this task involves a spatial and temporal dimension. In this paper, we present a visualization that supports analysts in monitoring events/topics and emotions both in time and in space. The visualization uses a clock face metaphor to encode temporal and spatial relationships, a color map to reflect emotion, and tag clouds for events and topics. A hierarchy of these clock faces supports drilling down to finer levels of granularity as well as rolling up the vast and fast flow of information. In order to showcase these functionalities of our visualization, we discuss several case studies that use the live data stream of the Twitter microblogging service. Finally, we demonstrate the usefulness and usability of the visualization in a user study that we conducted.
\end{abstract}

\section{Introduction and motivation}

In recent years, there has been a continuous develop ment of social media services on the web. As these services changed the way how people communicate with each other and their role in daily life increased, it was only a question of time until social media became a source for information gathering. In particular, there have been sev eral proposals to tap social media users as "social sensors" [19]. By analyzing user generated social media data, it is possible to gain unique insights into a wide variety of different topics from all over the world. Since a large amount of this data is generated by users on their mobile devices, it is often automatically enriched with their

\footnotetext{
* Corresponding author.

E-mail address: andreas.weiler@uni-konstanz.de (A. Weiler).
}

geographical location and other metadata about the user. This metadata opens up additional opportunities for the analysis of social media data. For example, it is possible to identify the incoming content information as a local report from an on site person.

Originally introduced in 2006 as a platform to exchange short messages ("tweets") on the Internet, Twitter has rapidly become one of the most popular social media platforms and an extremely influential channel for broadcasting news and a means of real time information exchange. With its currently about 302 million monthly active users who generate over 500 millions tweets daily, ${ }_{1}$ Twitter is not only an attractive communication channel, but has revolutionized the information exchange on the Internet and opened up new possibilities for knowledge

\footnotetext{
${ }^{1}$ https://about.twitter.com/company (May 22, 2015)
} 
acquisition from social interaction streamed in real time. Twitter encourages a large number of users to broadcast current news, situational statuses, personal matters, or feelings and ratings into the public sphere. The resulting data stream contains a high volume of live data consisting of all types of information from all over the world.

Unfortunately, the vast amount and the high variability in the quality of user generated data are obstructive to these analysis tasks. To address this problem, a large number of approaches for event and topic detection and tracking in social media data have been proposed. In pre vious work, we have focused on evaluating state of the art event detection techniques with respect to their task based and run time performance [28]. Additionally, we have designed a number of evaluation measures [27] that support the automatic evaluation of such techniques without the requirement of crafting ground truth data manually. In order to confirm the validity of our automatic measures, it has frequently been necessary to visualize and analyze a large number of events both in time and in space.

In this paper, we present a visualization that we initi ally designed to support these research efforts, but developed over time into a general tool for situation monitoring in user defined geographical areas. Motivated by our original requirements to analyze events/topics and emotions both temporally and spatially, our visualization uses a compact representation of time series event data based on a clock face metaphor. The temporal develop ment can be tracked based on successive time slices, each of which is spilt into two parts to track spatial relation ships. In this way, users can identify interesting data points inside a geographical region (filtered by geographical location tags) as well as about this region from anywhere else (filtered by keywords). A hierarchy of clock faces is used to support the roll up of the continuous event stream and the drill down to finer levels of granularity. By using multiple instances of the visualization, the evolution of events/topics and emotion can be tracked and compared among different urban areas over time.

This paper is an extended presentation of Weiler et al. [26], where we presented SiCi Explorer to detect and track emotions and stories in and around cities. With respect to the original paper, this paper makes the following new contributions. Section 2 describes several improvements to the visualization itself. We now use LDA [4] for topic modeling instead of simply displaying single terms and apply our previous work [25] to detect events in order to highlight them in the visualization. We assume that the combination of topics and important events generates a meaningful representation of the current reported situa tion. To avoid visual clutter in the visualization, we improved the term weighting and layout mechanism. Furthermore, we enriched the functionality of the appli cation by adding a detail view to the found topics and events, which directly shows the tweets for selected terms. Section 3 presents a series of new case studies that showcase the functionality of the visualization, whereas Section 4 discusses the results of a user study that was conducted specifically for this paper in order to demon strate the validity of our approach. Finally, we summarize related work in Section 5 and give concluding remarks in Section 6.

\section{System design}

The high volume and arrival rate of tweets makes it difficult for users to track the evolution of topics in the continuous data flow. Furthermore, it is challenging to discriminate between the normal behavior of the "social sensor" and unusual or abnormal behavior, which usually is an indicator for an interesting event in the area. There fore, our main goal is to provide a visualization for tracking the evolution of topics and emotions both in time and in space. For the latter, we distinguish between tweets that originate from within the geographical area that is ana lyzed and tweets that contain information about the area. To gather this situational information, we make use of Twitter users as a "social sensor". We combine a topic modeling and an event detection approach to extract not only ongoing topics, but also unexpected and important events in the corresponding data windows. This enables analysts, information seekers, or standard users to keep an overview of the massive stream of Twitter data, by mon itoring the aggregated topics and also in getting notified about interesting and unusual points in time by looking at the events.

\subsection{Processing pipeline}

The processing pipeline is built using Niagarino, ${ }^{2}$ a lightweight and multi threaded data stream management system. We have chosen Niagarino as an implementation platform as its modular architecture supports user defined operators, e.g., to perform sentiment derivation. Fig. 1 shows a detailed overview of the operators used to process the Twitter data stream for the proposed visualization. After scanning tweets from the data source, an operator based on a language detection library ${ }^{3}$ derives the lan guage and filters out non English tweets. The next opera tor tokenizes the tweet content. A set of "good tokens" is used to filter tweets further. This set only includes tokens, which are not contained in a common English stop word list, longer than a predefined token length, and do not contain characters that repeat more than two times, which is an indication of an unimportant term. Before executing the next steps, tweets are classified according to whether they originate from the analyzed area or whether they contain information about this area.

A tweet is classified as inside, if the coordinates of the geographical metadata of the tweet is inside a defined radius (in miles) around the defined center coordinates of the urban area. For this we use the Haversine [20] formula to calculate the great circle distance between the defined center coordinates and the current coordinates of the tweet. In contrast, a tweet is classified as outside, if the

\footnotetext{
${ }^{2}$ http://www.informatik.uni-konstanz.de/grossniklaus/software/ niagarino/

${ }^{3}$ http://code.google.com/p/language-detection/ (May 22, 2015)
} 
content of the tweet mentions one of the defined key words of the urban area, but has no coordinates that are inside the defined geographical area. Note, since not all Twitter users activated the enrichment of tweets with coordinates from their mobile device, some tweets may be wrongly classified as outside, even if the origin of the tweet is inside the area. After the classification of a tweet into inside, outside, or noise tweets, the stream is multi plexed into two parallel running phases shown in Fig. 1: topic and emotion aggregation (highlighted in blue) and event detection (highlighted in red). In the following, we describe each of the phases in more detail.

In the topic and emotion aggregation phase, the under lying data of the visualization is calculated. First, the sen timent value of each tweet is derived by applying an external library [22], which analyzes the text of the mes sage and returns values for the sentiment between 4 to

1 (extremely negative to negative), 0 (neutral), and 14 (positive to extremely positive). The next step segments the stream into tumbling windows of a predefined size $n$. For each of the resulting windows, the average of the sentiment values is calculated and the corresponding

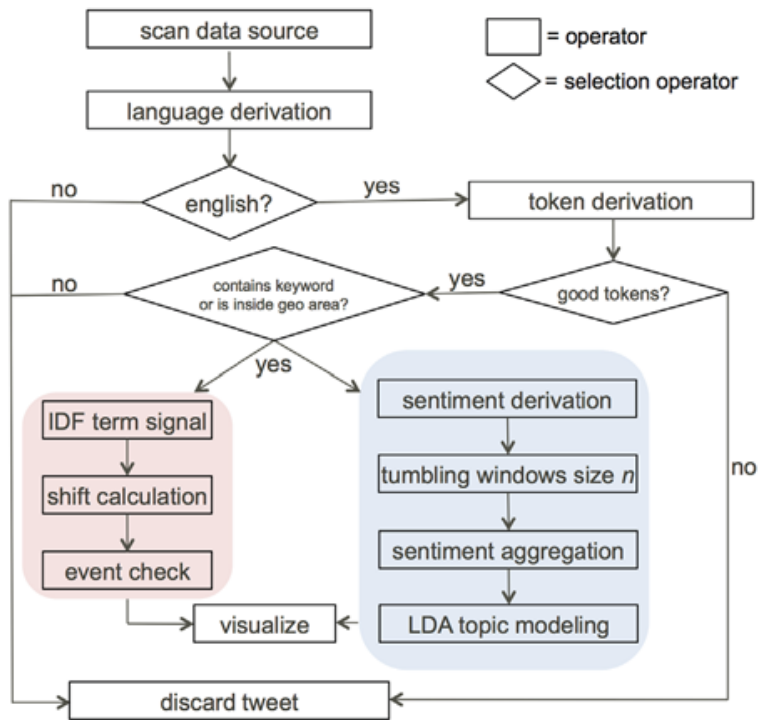

Fig. 1. Niagarino query plan to process the Twitter data stream. (For interpretation of the references to color in this figure caption, the reader is referred to the web version of this paper.) tweets are collected. For the collection of tweets in each window, topic modeling to create a predefined number of topics is performed using the Latent Dirichlet Allocation (LDA) implementation of McCallum [16]. For our case studies, we used LDA with 100 iterations to extract 10 topics with each of them consisting of five topic terms.

The event detection phase is based on our previous work [25] and enriches the extracted topics, with detected events, which are not likely to be contained in the set of topics. Additionally, we assume that the event terms express the importance of the terms in the corresponding time window. We use the inverse document frequency [21] of single terms to calculate how the frequency of terms changes over time. For this, shifts in the IDF are con tinuously calculated from time window to time window. The behavior of the resulting signal is considered abnor mal if a shift increases over a certain threshold for a pre defined number of time windows. In contrast to our pre vious work, we apply event detection to the two sub streams inside and outside, instead of to the full stream of tweets. If an event is detected, it is directly forwarded to the visualization module and placed at the corresponding position.

After completion of both phases, the results are merged together and the terms are weighted. Event terms, which result from the event detection phase, are weighted as the most important terms in the corresponding time window. If a topic term, which results from the topic modeling phase, also contains an event term, it is removed from the topic term list. The main topic term and the associated topic terms are weighted according to their rank computed by the $L D A$. The extracted data for emotion, topics, and events are then forwarded to the visualization module.

\subsection{Visualization}

In order to visualize the evolution of topics and emo tions inside and about an urban area over time, we use a hierarchical clock face metaphor. This metaphor allows the continuous ongoing evolution of topics, events, and emotions to be visualized. Fig. 2 shows the basic outline and the different hierarchy levels of the visualization. To differentiate between "inside" and "from outside" the area, each level is split into two parts. The inner circle visualizes the situation inside the chosen area, whereas the ring between the inner and outer circle visualizes the situation

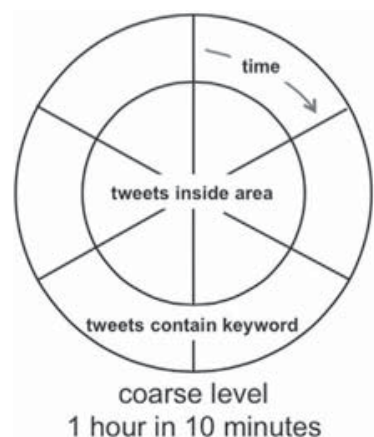

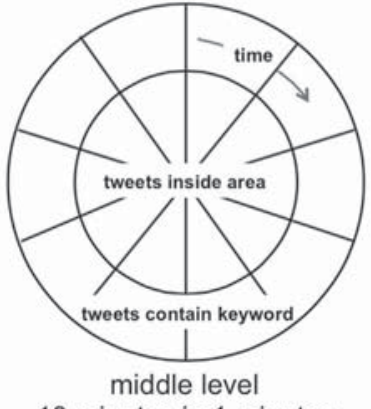

10 minutes in 1 minutes

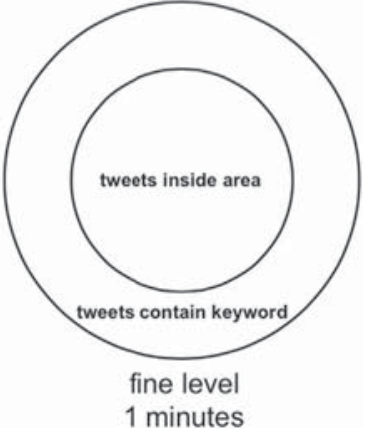

1 minutes

Fig. 2. Empty shapes showing the basic outline of the visualization. 
as perceived from outside the area. Topics and events are visualized using a shape conforming tag cloud. The tag cloud is adjusted to the shape of the corresponding time slice using an existing framework. ${ }^{4}$ Therefore, the ren dering of a single term cannot overlap different time sli ces, but the same term can repeatedly occur in more than one time slice. To increase the readability, all terms are aligned horizontally. Since events are a special form of topics, these terms are highlighted in purple to underline their importance. All terms are sized according to the weight assigned by the processing phase. The emotion of a time slice is visualized by shading its area with a color that displays the average of all sentiment values of tweets in the corresponding data window. To reflect the different levels of the sentiment, we use a color map from Color Brewer [11], which is shown at the bottom left of each visualization. On a scale from 0 to 255 , the opacity of these color values is set to 100 in order not to eclipse the underlying map.

Continuous updates to the visualization are supported based on three levels of granularity that we defined for the clock metaphor in order to track the evolution of topics and emotions over time. These levels respectively use $1 \mathrm{~min}, 10 \mathrm{~min}$, and hourly windows. As shown in Fig. 3, the three granularity levels are used by our visualization in two ways. First, the live visualization starts with $1 \mathrm{~min}$ windows, which are rolled up after $10 \mathrm{~min}$ to $10 \mathrm{~min}$ windows. These windows are again rolled up to hourly windows after $1 \mathrm{~h}$ of processing. In order to support events with arbitrary duration, this process repeats each hour for as long as the user chooses to monitor a city. Second, the zooming feature of the visualization uses these different granularity levels as visualization layers that enable the user to drill down and explore the data sets more deeply.

The coarse level of granularity (cf. top row of Fig. 6) displays the situation for a full hour. Since the next level visualizes $10 \mathrm{~min}$ of data, this level is split into six time slices of $10 \mathrm{~min}$ each. Each time slice summarizes the sentiment and the topics of the next level. Based on these summaries, it is possible to select interesting points in time that can then be explored on the next level in more detail. The middle level of granularity (cf. middle row of Fig. 6) displays the situation for a ten minute window. This layer is structured as the layer above, with the difference that it is split into 10 time slices of $1 \mathrm{~min}$ each. Finally, the fine level of granularity (cf. bottom row of Fig. 6) displays the situation for a one minute window. Since one design goal is the live observation of situations and we choose the update time of one minute as the finest granularity level.

The default background of the visualization is a colored OpenStreetMap ${ }^{5}$ map (cf. Fig. 6). This map supports the user in understanding how large the radius around the analyzed urban area is. Since single terms, even if they are in a group of topic terms, are sometimes not self expla natory, it is helpful to obtain additional context informa tion. Therefore, we extended the visualization with an overview of the tweets that correspond to these single

\footnotetext{
${ }^{4}$ http://wordcram.org/ (May 22, 2015)

5 http://www.openstreetmap.org/ (May 22, 2015)
}

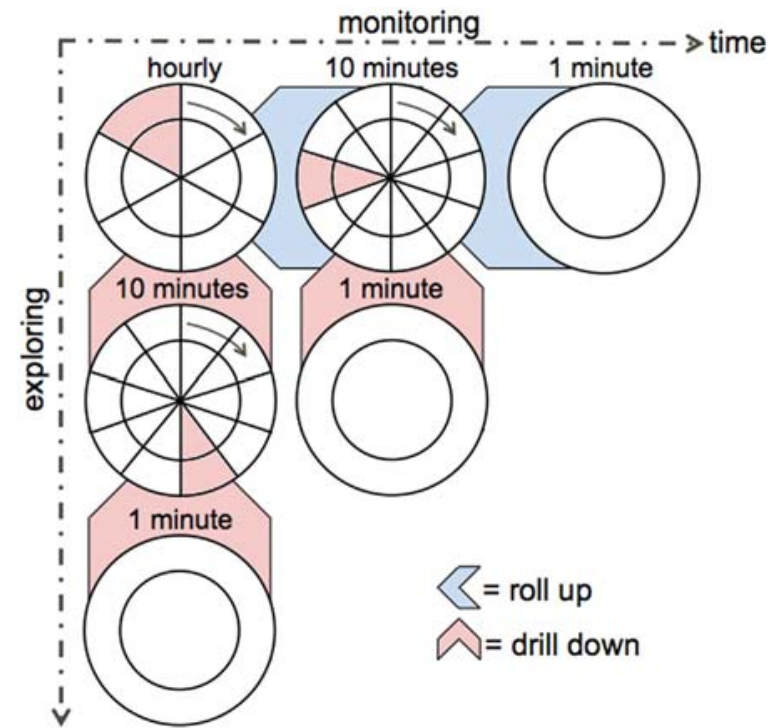

Fig. 3. Using roll up and drill down to support monitoring and exploring of events.

terms. For example, in Fig. 7 the selection of the term "shot" activates a popup window that displays the respective tweets. Hereby, it is possible to get deeper insight into the tweets that form the topic or event, both in terms of content (e.g., "Capitol Hill is locked down because of a gunman scared") and in terms of creation time. If desired, additional metadata (e.g., username, retweet count, geographical information) about the tweet can also be displayed.

\section{Case studies}

In order to demonstrate the generality of our approach, we present a series of case studies for different urban areas in the USA. For all case studies, we choose the name of the corresponding city as a filtering keyword for the tweets from outside as well as the center coordinate of the city with a predefined radius of 20 miles as limitation for the inside urban area.

\subsection{Twitter data set}

All of our case studies are based on data sets that we created from the live Twitter data stream. We use the Twitter Streaming $\mathrm{API}^{6}$ with the so called "Gardenhose" access level, which provides a randomly sampled sub stream containing $10 \%$ of the public live stream. By con ducting an exemplary evaluation of a representative sample of days, we can conclude that the incoming stream contains an average of over 2.5 million tweets per hour with an average of 35,000 tweets per minute. Since this type of stream only includes a very limited amount of tweets with geographic information, we merge the $10 \%$

\footnotetext{
${ }^{6}$ https://dev.twitter.com/ (May 22, 2015)
} 


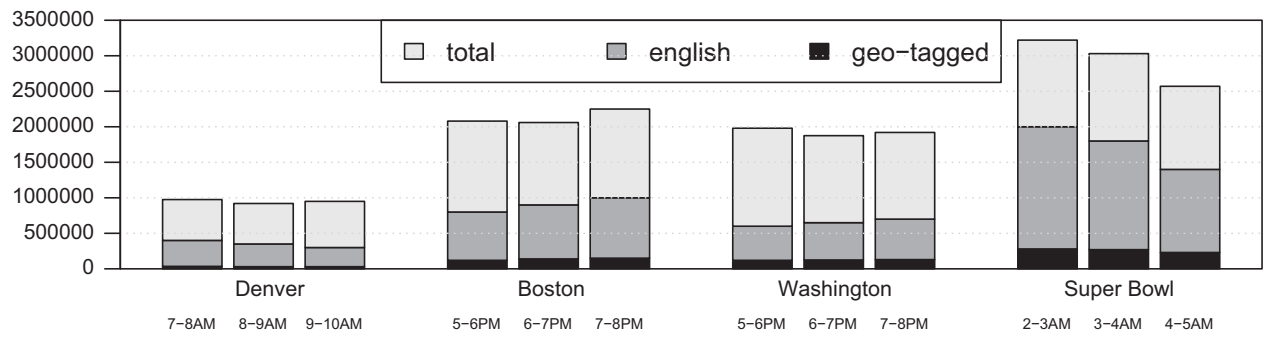

Fig. 4. Amount of total, English, and geo-tagged tweets per hour for each case study.

stream with five geographically filtered streams into a duplicate free stream of tweets. For these five geo graphically filtered streams, five bounding boxes are defined in terms of longitude and latitude values, which together cover the entire world. The $1 \%$ sample stream of Twitter is then filtered for additional tweets that are geo tagged and contained in one of these boxes. Therefore, the amount of tweets with location information in our data set is increased substantially. The geographic information either consists of the latitude and longitude values, which are automatically set by the used mobile device (over $80 \%$ of Twitter active users are mobile ${ }^{7}$ ) or a location manually added to the tweet by the author of the tweet message.

An overview of the data sets for the respective case studies is given in Fig. 4, which reflects the increasing amount of both total and English tweets starting with the Denver data set from 2012, to the Boston and Washington data sets from 2013, and to the Super Bowl data set from 2014. Also shown in the figure is the number of geo tagged tweets that is available in each data set. In the following, we report for each data set approximate numbers of tweets/hour that can be classified as from inside a mon itored city or as about a monitored city. In the Denver data set, 500 tweets/hour are from inside the city, whereas 1500 tweets/hour are about the city. In all other data sets, around 20004000 tweets/hour are from inside the monitored cities. The amount of tweets about the city in the Washington data set is roughly the same as in the Denver data set. Over the three hour period of the Boston data set, the amount of tweets about the city increases from 1500 to 290,000 tweets/hour. The Super Bowl data set is used to compare events in Seattle to events in Denver. There are 28,000 tweets in the first hour, 46,000 tweets in the second hour, and 27,000 tweets in the third hour that mention Seattle. In contrast, 37,000 tweets in the first hour, 65,000 tweets in the second hour, and 125,000 tweets in the third hour are about Denver.

These data sets were chosen for several reasons. First, they all contain an instance of an event that conforms to the definition [2] for which the visualization was designed. Second, as we will see in the following sections, each of these case studies allows a particular feature of the visualization to be showcased. Finally, as the four data sets have these different statistical properties, our visualization can be examined under different circumstances.

\subsection{Denver}

The first case study describes the observation of the urban area around the city of Denver on July 20, 2012 from $7 \mathrm{AM}$ to $10 \mathrm{AM}$ (UTC). Fig. 5 shows an overview of the chosen time frame. The color of the first hour reflects mostly positive emotions for the first $50 \mathrm{~min}$. However, in the last 10 min the emotions drift to mostly negative for the outside area. Terms like "mass", "shooting", and "aur ora" indicate that something has happened in that area. We can also see that these terms only appear from the outside and the emotion inside is still more positive. By looking at the tweets corresponding to "shooting", we can derive that there was a "Mass shooting in Aurora... at the Dark Knight Rises Premiere". In the second hour, events like "shooting", "killed", or "batman" appear. The emotions from outside turn to extremely negative. In the inside area, the emotions also turn to negative for the last $10 \mathrm{~min}$ of the hour. The third hour continues to display negative emotions for both areas.

In this case study, we can observe that the emotions and events are at first reported from outside about the area. A possible explanation for this is the low number of tweets in the defined area and the overall low rate of tweets in 2012. This demonstrates that the outside area is important to obtain quick notifications about situation changes, whereas the inside area is important to get on site reports about the situation from local people. We can conclude that by observing the evolution of topics and emotions, it is possible to recognize the ongoing event 2012 Aurora Shooting.

\subsection{Boston}

The second case study describes the observation of the city of Boston for $3 \mathrm{~h}$ on April, 15 2013. The top row of Fig. 6 shows hourly overviews for the hours from 5 PM to 8 PM (UTC). Most of the topic terms in the first hour are about the ongoing "marathon" in the city and also reflect a very positive emotion for within the urban area and from out side about the city. Terms like "running", "finish", "con grats", and "jeptoo" (name of the female winner) are shown, which indicates that some of the runners already finished the marathon. The event "desisa" (name of the male winner) between minutes 40 and 50 indicates that people celebrate the winner. The second hour also shows 

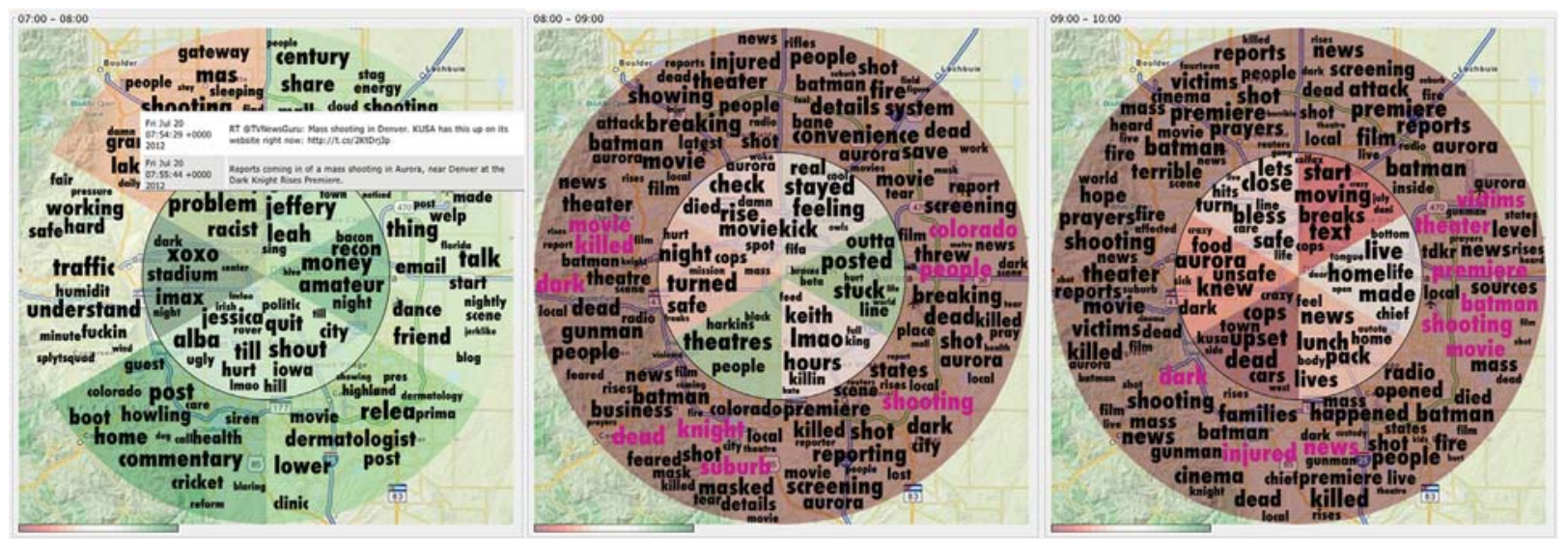

Fig. 5. Coarse level of the Denver case study from 7 AM to 10 AM (UTC) on July 20, 2012 from left to right. (For interpretation of the references to color in this figure caption, the reader is referred to the web version of this paper.)

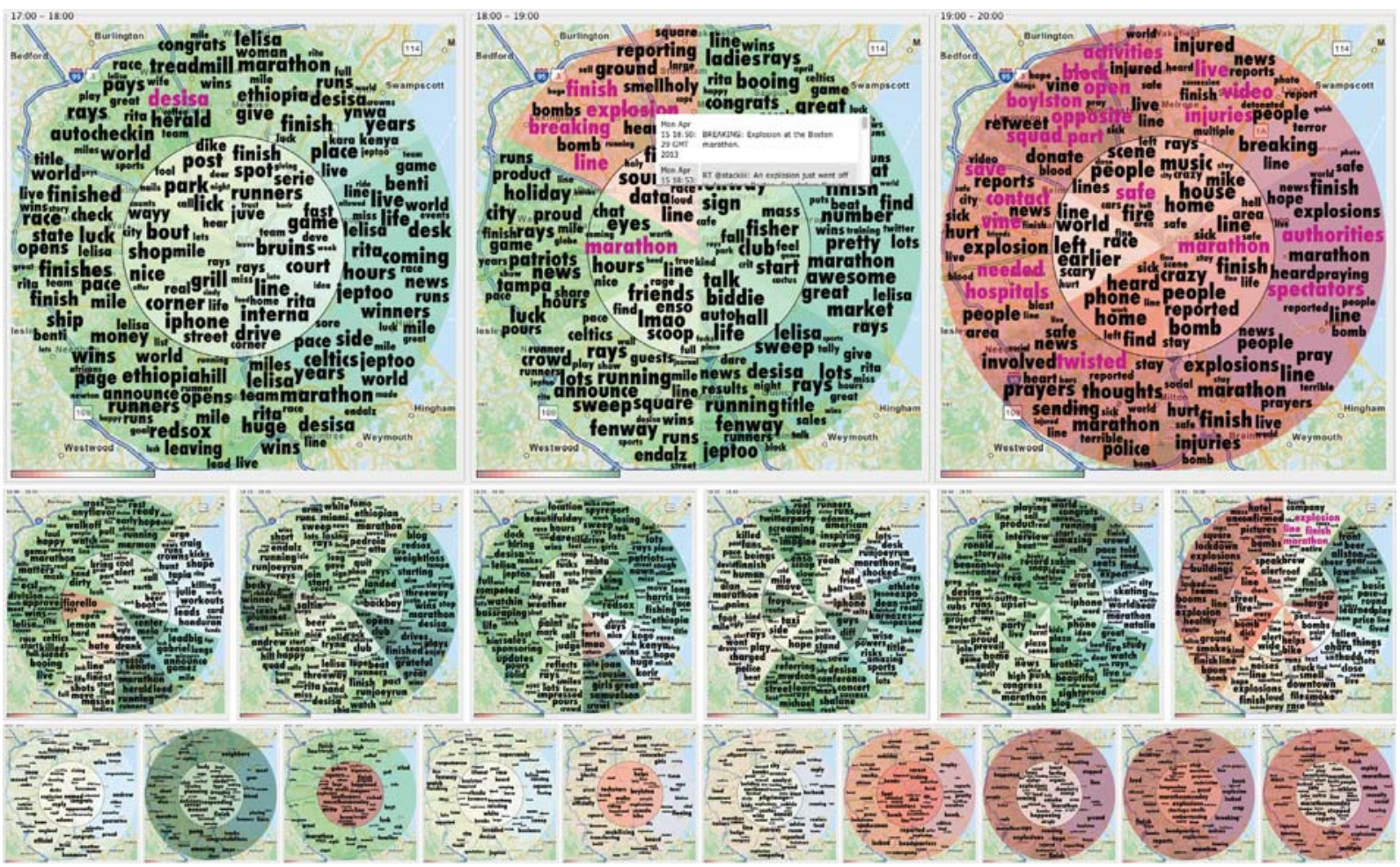

Fig. 6. Coarse level of the Boston case study from 5 PM to 8 PM (UTC) on April 15, 2013 from left to right (top), middle level from 6 PM to 7 PM (middle), and fine level from 6:50 PM to 7 PM (bottom). (For interpretation of the references to color in this figure caption, the reader is referred to the web version of this paper.)

very positive emotion for the first five segments of the hour. However, in the sixth segment, i.e., the last $10 \mathrm{~min}$ of the hour, the emotion drifts to negative for both areas. Also, the event "explosion" can be seen in the outside part and the topic term "bomb" can be seen in the inside part. By choosing the event term "explosion", more context information can be obtained in order to understand what has happened (e.g., "BREAKING: Explosion at the Boston Marathon"). Also, by checking out the term "coply" in the inner area we can discover a message that contains a hyperlink to an image, which actually shows one of the first on site pictures of the event.

Since the second hour shows this unexpected behavior and we are further interested in the unfolding of the situation, we drill down into the second hour of the visualization. The middle row of Fig. 6 shows the zoomed visualization with the second hour in 10 min windows. In this more detailed view, we can see that the term 

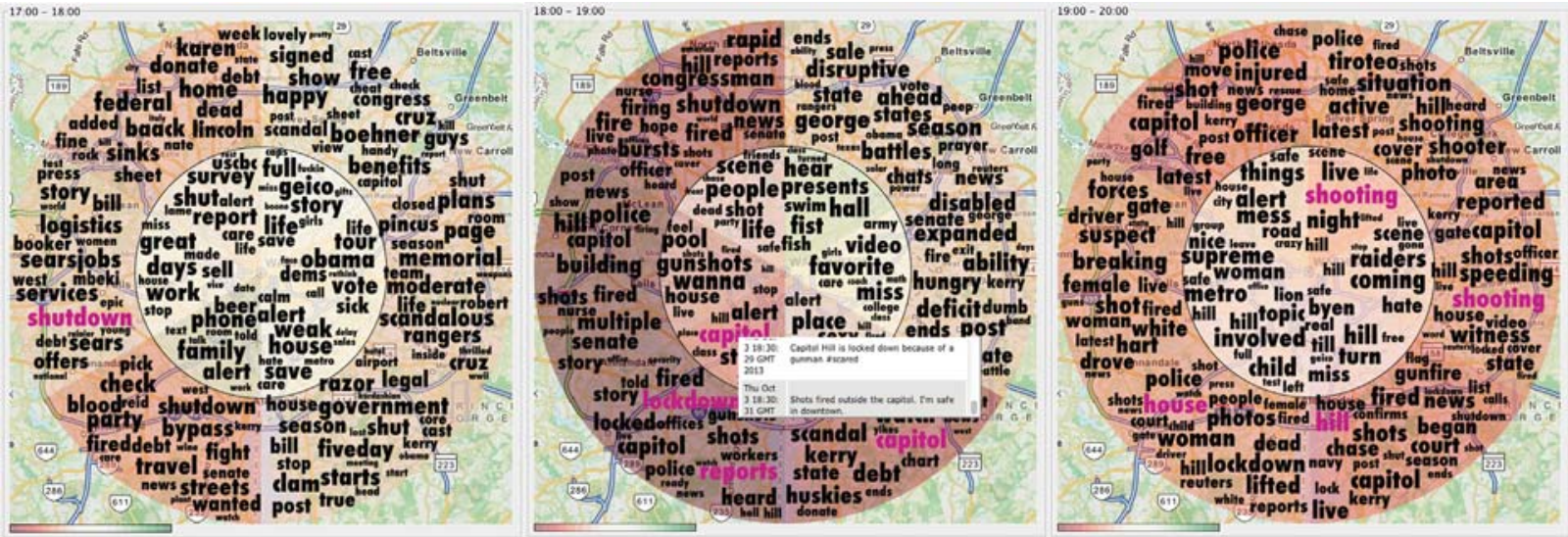

Fig. 7. Coarse level of the Washington case study from 5 PM to 8 PM (UTC) on October 3, 2013 from left to right.

"marathon" is always very frequent and forms an event twice. However, in the last 10 min of the hour the events "explosion", "breaking", and "line" take the lead as most important terms. After analyzing the middle layer of the visualization, we are further interested in the develop ments during the last $10 \mathrm{~min}$ of the second hour. There fore, we drill down into the last panel of the visualization. The bottom row of Fig. 6 shows the zoomed visualization of the 1 min windows. Here, we can identify that the term "explosion" appears in both areas of the second minute. Nevertheless, the emotion is still positive at that time, because the event has not spread widely yet. By looking at the following hour, we can notice that the negative emo tion increases strongly in both parts. Additional events like "injuries", "spectators", and "authorities" further indicate that something bad happened in Boston. An interesting observation is that the terms "prayers" and "thoughts" are more frequent from the outside, which is an indication that they are directed at the people living in the city.

We can summarize that the Boston Marathon Bombings ${ }^{9}$ can be identified using our visualization based on topic and event terms only 2 min after the event took place. Drilling down to finer levels of detail, the initial point for the event can be traced. While the emotion changes only slightly in the first couple of minutes, the negative emo tion increases substantially later and therefore gives a good indication of the extent of the tragedy.

\subsection{Washington}

The third case study describes the observation of the city of Washington on October 3, 2013 from 5 PM to 8 PM (UTC). Fig. 7 shows an overview of all $3 \mathrm{~h}$ in the chosen time frame. The negative emotions from outside reflect the overall dissatisfaction with the government in Washing ton, which is exemplified by terms like "scandal", "shut down", and "government". In the first hour, the emotions inside the city are neutral or slightly negative, but the term "alert" can be seen clearly in the tag cloud. An examination

\footnotetext{
${ }^{9}$ http://en.wikipedia.org/wiki/Boston_Marathon_bombings （May
} $22,2015)$ of the corresponding tweets reveals that they are all about advertising and therefore this term can be ignored. The second hour shows a clear change to negative emotions in the second half of the hour for both the city and sur rounding area. Additionally, the terms "fired", "gunshots", and the event "capitol" appear. By studying the tweets corresponding to this event, we can see that these reports (e.g., "Capitol Hill is locked down because of a gunman scared") are about the ongoing United States Capitol Shooting. ${ }^{10}$ The emotions of almost the whole third hour remain negative for inside the city and from outside about the city.

For this case study, we can summarize that the emo tions turned from slightly negative to extremely negative, instead of from extremely positive to extremely negative as in the first case study. We can also conclude that the emotion inside an area can be very different from the one perceived outside, which further motivates the spatial separation used in our visualization. Finally, the single tweets detail view can support users in clarifying whether a conspicuous term in a tag cloud (e.g., "alert") indicates that something interesting is happening or not.

\subsection{Super Bowl 2014}

The final case study presents a comparison between two cities during the "Super Bowl XLVIII", which was played between the Seattle Seahawks and the Denver Broncos. Therefore, we observe both cities on February 3, 2014 from 2 AM to 5 AM (UTC) (last $2 \mathrm{~h}$ of the game and $1 \mathrm{~h}$ afterwards). Fig. 8 shows the $3 \mathrm{~h}$ for both cities, with Seattle at the top and Denver at the bottom. There is almost no difference in emotions during the first hour. Both cities are more or less neutral or slightly positive tempered. Also, both cities talk about terms like "half time", "defense", and the names of the teams. This indi cates that both cities are engaged in the ongoing sports event. The second hour shows a substantial increase in positive sentiment for Seattle in the last $10 \mathrm{~min}$. In this

\footnotetext{
${ }^{10}$ http://en.wikipedia.org/wiki/United_States_Capitol_shooting_inci dent_(2013) (May 22, 2015)
} 

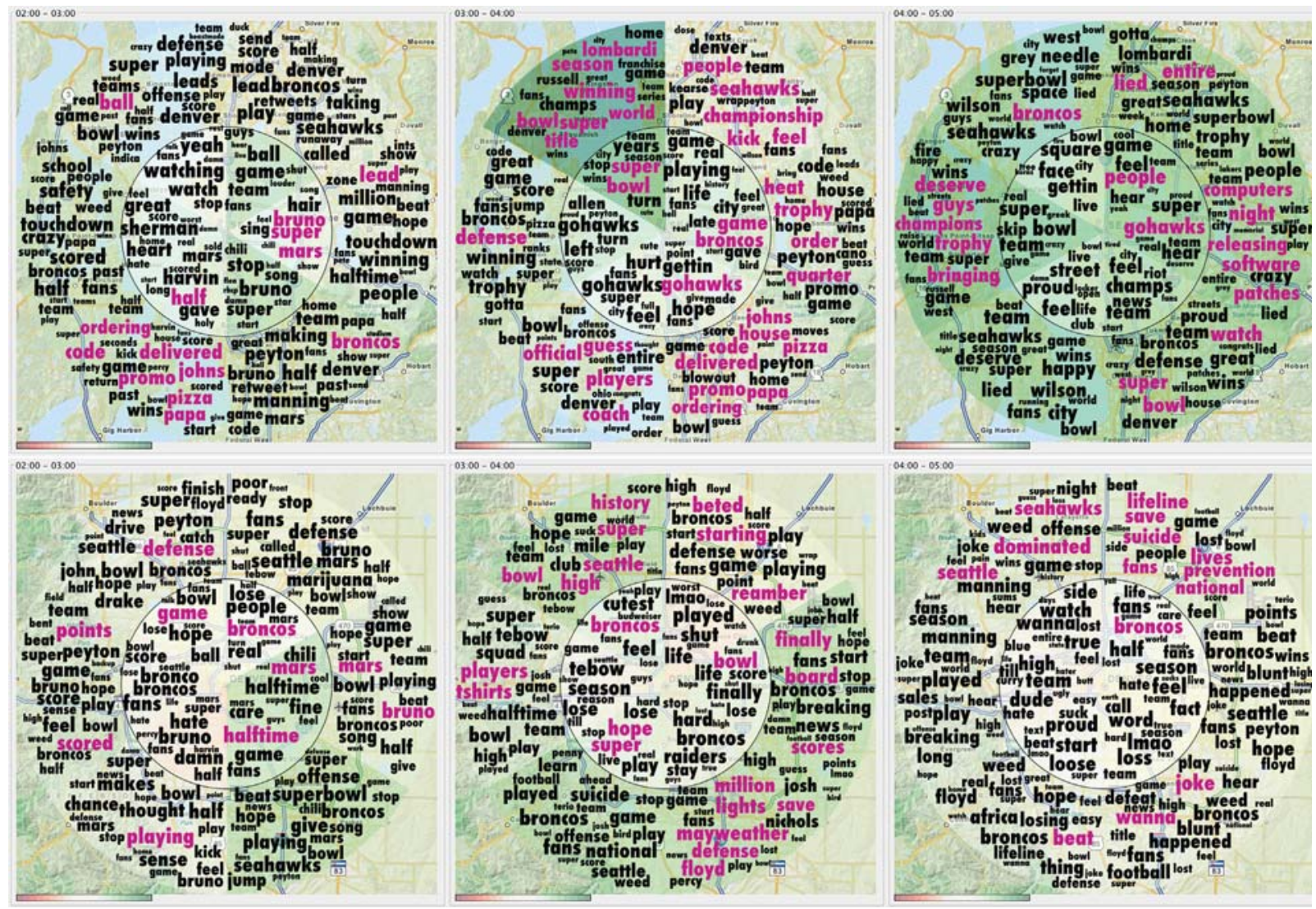

Fig. 8. Coarse level of the Super Bowl case study from 2 AM to 5 AM (UTC) on February 3, 2014 from left to right. Comparison of the cities Seattle (top) vs. Denver (bottom).

time slice, the game ended and the Seattle Seahawks won. At the same time, event terms like "winning" and "title" appear in the Seattle tag clouds for the last $10 \mathrm{~min}$. In Denver, the sentiment remains the same over the second hour. By studying the tweets corresponding to the event terms "high" and "history", we can derive that the 43:8 result was one of the highest defeats in history. The sen timent of the third hour clearly indicates that Seattle won the Super Bowl. While the sentiment in Seattle turns to positive and even to extremely positive, the sentiment in Denver turns to neutral.

This case study presents a comparison between two cities for an event, which is not happening in either one of them, but leads to much discussion and reactions from both sites. Although the sentiment for the losing city is not turning completely into negative, we can still recognize the winning city of the sports event. A possible reason for this lack of negative emotion might be the fact that people are less inclined to tweet about their team in case of a loss than in case of a win.

\section{User study}

In order to verify the usefulness and usability of the proposed visualization, we have conducted a web based user study. ${ }^{11}$ In particular, the goal of the study was to address the following four research questions:

1. Can users identify the topic of an event based on the visualization?

2. Can users recognize the sentiment associated with an event?

3. Can users track the development of an event using the visualization?

4. Can users interpret the spatial information that is visualized?

While each of these questions enables us to study one specific design choice, together they cover the full spec trum of analyses that we intend to support with our visualization. For every question, we have designed an experiment consisting of one ore more tasks that we asked participants of our study to perform. In the following, we first discuss these tasks in more detail and then present the results obtained from the user study.

\footnotetext{
${ }^{11}$ https://www.soscisurvey.de/sici/ (May 22, 2015)
} 


\subsection{Study design}

The overall study was structured in a prologue, four experiments corresponding to the four research questions, and an epilogue. The prologue consisted of a page that provided a short overview of what information is visua lized by our approach. With a probability of $50 \%$, users were then shown an additional page that explained to them how our approach visualizes this information, similar to Fig. 2. The epilogue was used to obtain feedback about the overall user experience as well as information about the users themselves, which they could optionally provide. Apart from age and gender, we asked users about their technical background and whether they have ever been diagnosed with daltonism, since our visualization heavily relies on a red green color profile to visualize sentiment.

The tasks to study the four research questions given above were designed as follows. For the first question, users were shown a visualization of an hour of data from one of the case studies introduced in the previous section. For each of these visualizations, we provided users a choice of three possible headlines as well as a "None of the above" option. The headlines were assembled by searching the web for news articles using the terms that appeared most frequently in the visualization. For example, we used the rightmost visualization shown in Fig. 5 together with the headlines "For the president, a Batman connection", "Dark Knight finale leaves Warner to reboot new Batman", and "Colorado movie theater shooting", where the last one describes the visualized event. In total, we created seven such tasks for participants to perform. For the second research question, we again showed participants six examples of our visualization. For each example, partici pants were asked to rate the visualized sentiment on a five point scale ranging from very positive to very nega tive. In order to study the third research question, we designed two different tasks. In the first task, participants were shown examples of our visualization for three dif ferent events. For each of these examples, participants were asked to indicate if an event happened during the visualized hour and, if so, in which of the six $10 \mathrm{~min}$ slices it most likely began. In the second task of this experiment, participants were shown three visualizations correspond ing to three consecutive hours of the same event. As the examples were not given in chronological sequence, we asked participants to put them in the correct order. In order to study the last question, participants were shown four examples of the visualization. For each example, we asked them to answer the following three questions on a five point scale ranging from strongly agree to strongly disagree.

Q1 The topic of the event inside the city is the same as elsewhere.

Q2 The sentiment of the event inside the city is the same as elsewhere.

Q3 The event develops in the same way inside the city as elsewhere.

Note that each of these questions revisits one of the previously studied aspects of our visualization, i.e., topic, sentiment, and development, which are now examined with respect to their spatial relationships.

\subsection{Results}

The user study was conducted with a total 67 partici pants. However, the results presented in this section are based on 54 participants only. We discarded eight parti cipants because they spent less than 5 min to complete the survey. Since all tasks except for the first one rely on par ticipants being able to differentiate red and green, another five participants were only included in the results of the first task, because they stated that they have been diag nosed with daltonism. The average age of participants was 23 years and $26 \%$ of them were female. $59 \%$ stated that they have a background in computer science, $11 \%$ in visualization, and $15 \%$ in data analysis. On average, the questionnaire took $15 \mathrm{~min}$ to complete.

In the first experiment that studied topic recognition, a total of $61 \%$ of the participants was able to identify the headline that correctly describes the visualized event. Participants that were previously given an explanation of our visualization performed better (66\%) than people without this instruction (58\%). However, the difference is not statistically significant ( $t$ test) with $p<0.1$. Given the similarity of the three headline options, this result clearly demonstrates that users are capable of identifying which real world event is visualized. Furthermore, the terms that our visualization displays are generated by simple LDA topic modeling. Due to the modularity of our approach, more advanced event detection techniques could be inte grated to further improve this result.

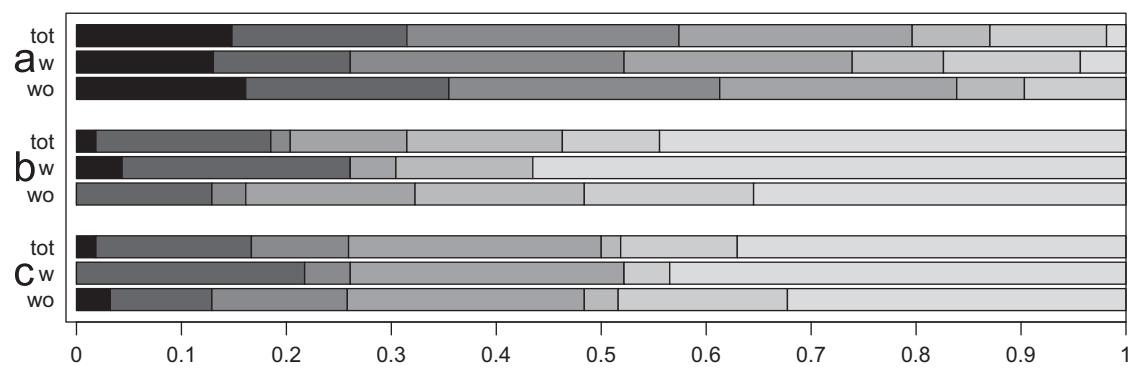

Fig. 9. Ratio of participants who identified an event in time-slice: never, 10, 20, 30, 40, 50, or 60 min (from dark to light). 

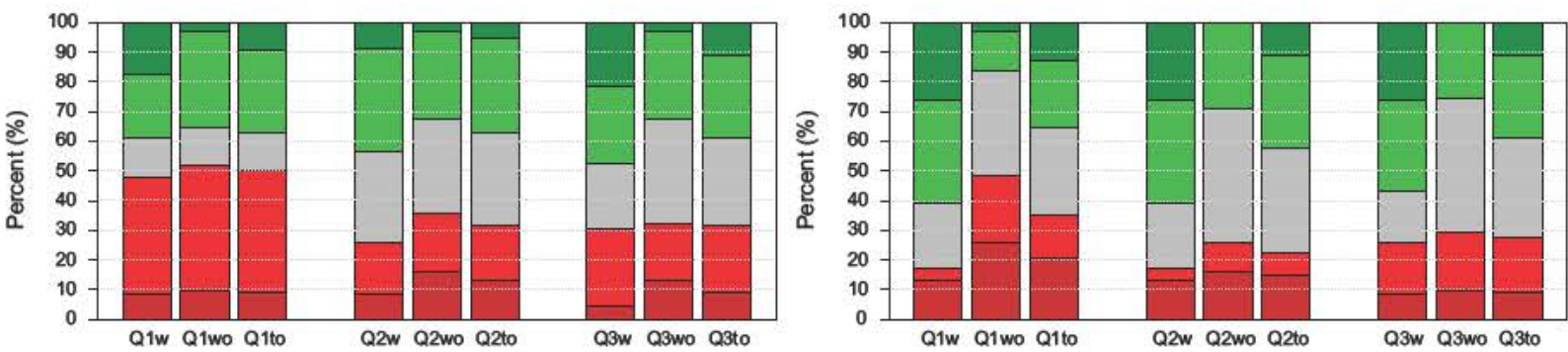

Fig. 10. Results of the experiment studying visualization of spatial information for the first (left) and second (right) hour of the Boston case study.
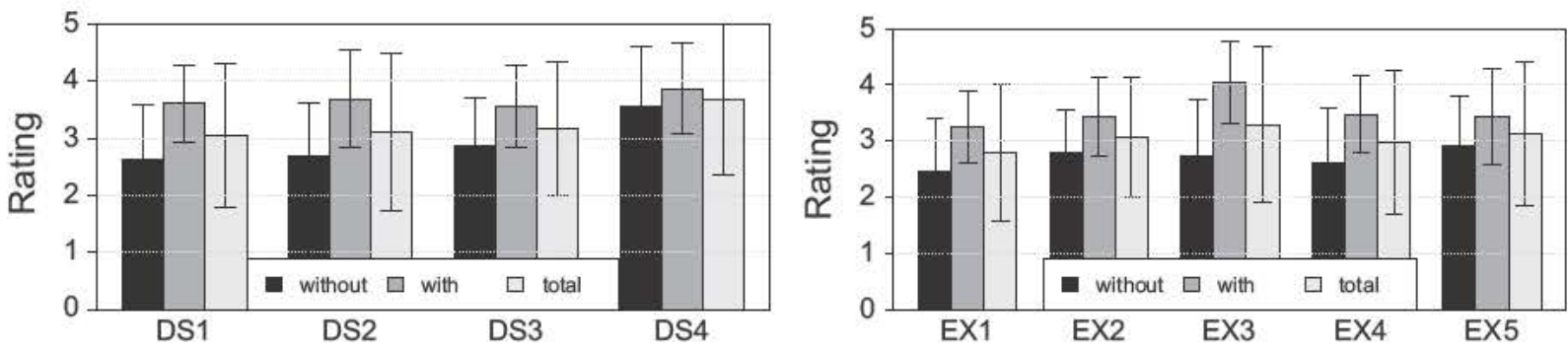

Fig. 11. Participant feedback on visualization design (left) and user experience (right).

$70 \%$ of all participants correctly classified the sentiment of an event in the second experiment studying sentiment recognition. Again, participants with prior instruction performed better (72\%) than participants without (68\%). Again, this difference is not statistically significant ( $t$ test) with $p<0.4$. In order to score answers the numerical sentiment values were mapped to the five point scale used in the study. Direct matches contributed fully to the score, while adjacent matches contributed half. Based on this result, we conclude that our visualization supports users in understanding the sentiment associated with an event. As with event topics, sentiment detection relies on a third party module that could be replaced to further improve results. We note that people suffering from red green blindness could not correctly identify sentiments and, therefore, consider an option to use an alternative color scale as future work.

For the first task of the third experiment, we used the following three visualizations: (a) Denver, $3 \mathrm{AM}$ to $4 \mathrm{AM}$ (Fig. 8, bottom center), (b) Boston, 6 PM to 7 PM (Fig. 6, top center), and (c) Seattle, $3 \mathrm{AM}$ to $4 \mathrm{AM}$ (Fig. 8, top center). The corresponding results are shown in Fig. 9. We have chosen to include visualization (a) in the user study as it is an example with very little fluctuation in both event topics and sentiments over the six $10 \mathrm{~min}$ slices. As a con sequence, it is difficult to identify a single point in time at which an event is happening. The results of the user study confirm this expectation as no single time slice is selected consistently by all users. Also, only $15 \%$ of the participants indicated that they could not detect an event at all. The time slices selected by the remaining users show a bias towards the 20 and 30 min slice. Revisiting the visualiza tion, this choice can be explained by the change in senti ment (from the first to the second time slice) and by the increase of highlighted terms (from the second to the third time slice). In contrast to this first example, a detectable event was actually happening in the sixth time slice of visualizations (b) and (c). The results of our user study clearly reflect this as a majority of users is able to select the correct time slice in both cases. Again, we can observe that users with prior instruction perform better than users without. However the difference is not statistically sig nificant ( $t$ test) with $p<0.2$ for (b) and $p<0.5$ for (c). In the second task of this experiment studying event devel opment, a total of $66 \%$ of the participants selected the correct ordering of visualizations out of all six possible orderings. An unexpected outcome of this second task is that participants without instruction performed better (73\%) than average. Based on these results, we conclude that our visualization can support users in detecting and tracking events. However, we also acknowledge that there is room for improvement in this aspect of our visualiza tion. As a first step into this direction, we plan to conduct targeted interviews with participants who did not wish to stay anonymous to better understand the reasoning for their choices in these tasks during the user study.

For reasons of space, we limit the discussion of the results of the fourth experiment to the examples taken from the Boston case study. Fig. 10 plots the answers to the three questions from the previous section for the visuali zation of the first and the second hour of the event (cf. Fig. 6, left and middle). First of all, we note that partici pants who received the initial explanation of our visuali zation performed better at the task they were given in this experiment. In particular during the second hour, the dif ference is statistically significant ( $t$ test) for $\mathbf{Q 1}(p<0.002)$, Q2 $(p<0.04)$, and Q3 $(p<0.04)$. Therefore, we focus on the results labeled with " $w$ " in the figure. Recall that the dominating event happened towards the end of the sec ond hour, while a variety of topics was present during the 
first hour. Accordingly, a majority of participants answered that the event topics (Q1) inside the city are different from the ones from outside during the first hour. However, a majority of participants was also able to determine that the perception of the event topics from inside the city aligned with the one from elsewhere as tweets about the attacks started to dominate in the second hour. The same effect can also be observed for the question about senti ment perception $(\mathbf{Q 2})$. During the first hour, the sentiment of tweets about Boston is slightly more positive than the sentiment of tweets originating in Boston, which explains the few negative answers. Again, this changes considerably in the second hour as reflected by an increase of positive answers. The same effect is less pronounced for the last question about perception of event development (Q3). As little is happening during the first hour, the visualization inside and outside Boston is very similar, which is reflected in the relatively high percentage of positive answers. The same is true for the development of the actual event in the second hour, which explains the only slightly increased percentage of positive answers. Based on these results, we conclude that expert users or trained users can gain a understanding of how event topics, sentiments, and development are perceived in different places. However, the results also show that this aspect of our visualization is not intuitively accessible to novice or untrained users.

Finally, Fig. 11 plots the answers to the feedback ques tions from the epilogue of the questionnaire. On the left, we asked participants to rate the following design ele ments of our visualization: spatial separation (DS1), clock face metaphor (DS2), term clouds (DS3), and color scheme (DS4). Participants generally responded favorably to our design choices, with approval being higher among users who received an explanation of the visualization. On the right, questions were targeted at the user experience in terms of ease of understanding the overall situation at a glance (EX1), identifying event topics (EX2), grasping the sentiment of events (EX3), comparing the perception of events inside and outside the analyzed city (EX4), and tracking the development of events (EX5). User feedback ranged from neutral to slightly positive, with better scores from participants who were told how to use the visualization.

\section{Related work}

A lot of research has been done on analysis and knowledge discovery from social media data. As a good overview, the survey [5] about sense making of social media data lists state of the art approaches for mining semantics from social media streams. The use of social media data for the analysis or exploration of information about cities or local areas is for example presented in "CityBeat" [30] and "Whoo.ly" [12]. From a stream of geo tagged photos from Instagram, "CityBeat" extracts what is going on in a city and alerts users to unusual activities. "Whoo.ly" extracts and summarizes hyperlocal informa tion about events, topics, people, and places from Twitter posts. In contrast to our work, they have the possibility to use the 100\% Firehose access of Twitter to gather the full public stream. Furthermore, they do not provide a visua lization or integrate sentiment information in their appli cation. Other research projects examine the evolution of user communities in social media. For example, Giatsoglou et al. [10] present a work on the evolution and change of user communities during events by using microblog data. In the "Livehoods" project [7], Foursquare checkins are clustered in order to identify the living areas of people. To evaluate this approach, face to face interviews with citi zens of Pittsburgh were conducted, which revealed that their answers matched the results of the technique.

Because of the fast propagation speed of information in social media networks, a lot of research focuses on event or topic detection and tracking [2] for various domains. For example, Sakaki et al. [19] present a system for earthquake detection and [29] a system to detect events during elec tions in the Twitter data stream. In addition to domain specific systems, open domain event detection systems, like "TwitInfo" [15], "enBloque" [3], and "TwiCal" [18] have also been developed. Further research is undertaken in the area of epidemics tracking [8] and disaster [13] or crisis [17] management. Aiello et al. [1] also use "social sensors" to sense trending topics by applying different techniques to the Twitter social data stream.

The survey of Wanner et al. [24] presents different visual analysis methods for event detection in text data streams. However, no visual analysis method is mentioned that supports the temporal and spatial analysis of events based on urban areas. Nevertheless, there are a number of works that emerged in the area of visual analytics for Twitter social data streams. For example, "SensePlace2" [14] supports overview and detail maps of tweets, place time attribute filtering of tweets, and analysis of changing issues and perspectives over time and across space as reflected in tweets. However, in contrast to our work, they use a crawler to systematically query the Twitter API for tweets containing any topics deemed to be of interest, instead of using the data stream directly. "ScatterBlogs2" [6] is another approach that lets analysts build task tailored message filters in an interactive and visual man ner based on recorded messages of well understood pre vious events. In contrast to our work, it is possible to create more powerful filters and also to redefine them. However, they do not provide an overview visualization to track the evolution of topics and events over time. Fur thermore, they do not include any information about emotions. Another work is presented by Dork et al. [9], which is dubbed "a visual backchannel for large scale events". The authors present a novel way of following and exploring online conversations about large scale events using interactive visualizations based on a timeline. "Sen timentClock" [23] is an approach that visualizes daily timelines of tweets using a clock face metaphor. The fre quency of tweets is shown in different categories by clas sifying them according to sentiment (e.g., deactivation, pleasant, activation, and unpleasant) and time of the day (e.g., morning, afternoon, evening, and night). However, both these works do not provide any information about geographical characteristics of the tweets. Another differ ence is that they predefine the tweet set by a collection of keywords or hashtags. 


\section{Conclusions}

In this paper, we presented a visualization that can support analysts in tracking events in terms of topics and sentiments both over time and in space. Specifically, the situation in urban areas as reflected by the geo tagged tweets in the live social media data stream of Twitter can be monitored. As a consequence of this approach, our visualization leverages the growing number of social media users as "social sensors" to obtain information from within the monitored area as well as from elsewhere about the monitored area. The visualization is based on a clock face metaphor that is used to represent both temporal and spatial developments. A hierarchy of these clock faces supports user interaction by means of drilling down to finer levels of granularity or rolling up the incoming event data stream to gain an overview.

Our case studies showed in detail how the visualization supports these tasks. Furthermore, the case studies docu mented the need for all of the functionality provided by the visualization. For example, the temporal tracking of event topics and sentiments was showcased by the Boston case study, whereas the separation of the visualization was motivated by the Washington case study. The Denver case study demonstrated the usefulness of separating the visualization in two parts to track the development of events inside the monitored area independent from the development elsewhere. Finally, the Super Bowl case study showed how multiple instances of the visualization can be used to compare the development of the same event in different areas.

Our detailed user study demonstrated that expert or trained users are indeed capable of applying the visuali zation in order to perform these analysis tasks. However, it has also shown that some aspects of the visualization are not intuitive to novice or untrained users. We do not see this as a limitation of our visualization as it was originally designed with advanced users in mind. Furthermore, detecting and tracking events is itself a very challenging task. However, we do believe that some of the results in the user study can be improved by integrating more advanced event detection and sentiment derivation tech niques into our approach. Due to the use of the modular and extensible Niagarino data stream management sys tem, this can be achieved in future work with little effort.

\section{Acknowledgements}

The authors would like to thank the anonymous reviewers and Martin Brunner for their valuable feedback that greatly helped to improve the quality of this paper.

\section{References}

[1] L.M. Aiello, G. Petkos, C. Martin, D. Corney, S. Papadopoulos, R. Skraba, A. Göker, I. Kompatsiaris, Sensing trending topics in twitter, IEEE Trans. Multimed. 15 (6) (2013) 1268-1282.

[2] J. Allan, Topic Detection and Tracking: Event-based Information Organization, Kluwer Academic Publishers, Norwell, MA, USA, 2002.
[3] F. Alvanaki, S. Michel, K. Ramamritham, G. Weikum, See what's enBlogue: real-time emergent topic identification in social media, In: Proceedings of the International Conference on Extending Database Technology (EDBT), 2012, pp. 336-347.

[4] D.M. Blei, A.Y. Ng, M.I. Jordan, Latent Dirichlet allocation, J. Mach. Learn. Res. 3 (2003) 993-1022.

[5] K. Bontcheva, D. Rout, Making sense of social media streams through semantics: a survey, Semant. Web 5 (5) (2014) 373-403.

[6] H. Bosch, D. Thom, F. Heimerl, E. Puttmann, S. Koch, R. Krüger, M. Wörner, T. Ertl, ScatterBlogs2: real-time monitoring of microblog messages through user-guided filtering, IEEE Trans. Vis. Comput. Graph. 19 (12) (2013) 2022-2031.

[7] J. Cranshaw, R. Schwartz, J.I. Hong, N.M. Sadeh, The livehoods project: utilizing social media to understand the dynamics of a city, In: Proceedings of the International Conference on Weblogs and Social Media (ICWSM), 2012.

[8] A. Culotta, Towards detecting influenza epidemics by analyzing twitter messages, in: Proceedings of the International Workshop on Social Media Analytics (SOMA), 2010, pp. 115-122.

[9] M. Dork, D. Gruen, C. Williamson, S. Carpendale, A visual backchannel for large-scale events, IEEE Trans. Vis. Comput. Graph. 16 (6) (2010) 1129-1138.

[10] M. Giatsoglou, D. Chatzakou, A. Vakali, User Communities Evolution in Microblogs: A Public Awareness Barometer for Real World Events. World Wide Web, 2014

[11] M. Harrower, C.A. Brewer, ColorBrewer.org: an online tool for selecting color schemes for maps, Cartograph. J. 40 (1) (2003) 27-37.

[12] Y. Hu, S.D. Farnham, A. Monroy-Hernández, Whoo.ly: facilitating information seeking for hyperlocal communities using social media, in: Proceedings of the International Conference on Human Factors in Computing Systems (CHI), 2013, pp. 3481-3490.

[13] S. Lee, S. Lee, K. Kim, J. Park, Bursty event detection from text streams for disaster management, In: Proceedings of the International Conference on World Wide Web (WWW) (Companion Volume), 2012, pp. 679-682.

[14] A.M. MacEachren, A.R. Jaiswal, A.C. Robinson, S. Pezanowski, A. Savelyev, P. Mitra, X. Zhang, J. Blanford, SensePlace2: GeoTwitter analytics support for situational awareness, In: Proceedings of the International Conference on Visual Analytics Science and Technology (VAST), 2011, pp. 181-190.

[15] A. Marcus, M.S. Bernstein, O. Badar, D.R. Karger, S. Madden, R.C. Miller, Twitinfo: aggregating and visualizing microblogs for event exploration, in: Proceedings of the International Conference on Human Factors in Computing Systems (CHI), 2011, pp. 227-236.

[16] A.K. McCallum, MALLET: A Machine Learning for Language Toolkit, URL 〈http://mallet.cs.umass.edu 〉, 2002.

[17] S.E. Middleton, L. Middleton, S. Modafferi, Real-time crisis mapping of natural disasters using social media, IEEE Intell. Syst. 29 (2) (2014) 9-17.

[18] A. Ritter, O. Mausam Etzioni, S. Clark, Open domain event extraction from twitter, In: Proceedings of the International Conference on Knowledge Discovery and Data Mining (SIGKDD), 2012, pp. 1104-1112.

[19] T. Sakaki, M. Okazaki, Y. Matsuo, Earthquake shakes twitter users: real-time event detection by social sensors, In: Proceedings of the International Conference on World Wide Web (WWW), 2010, pp. 851-860.

[20] R.W. Sinnott, Virtues of the haversine, Sky Telesc. 68 (2) (1984) 159.

[21] K. Spärck Jones, A statistical interpretation of term specificity and its application in retrieval, in: Document Retrieval Systems, Taylor Graham Publishing, London, UK, 1988, pp. 132-142.

[22] M. Thelwall, K. Buckley, G. Paltoglou, D. Cai, A. Kappas, Sentiment strength detection in short informal text, J. Am. Soc. Inf. Sci. Technol. 61 (12) (2010) 2544-2558.

[23] F.Y. Wang, A. Sallaberry, K. Klein, M. Takatsuka, Visualizing timevarying twitter data with sentimentclock (Poster), In: Proceedings of the International Conference on Information Visualization (InfoVis), 2014.

[24] F. Wanner, A. Stoffel, D. Jäckle, B.C. Kwon, A. Weiler, D.A. Keim, Stateof-the-art report of visual analysis for event detection in text data streams, iln: R. Borgo, R. Maciejewski, I. Viola (Eds.), EuroVis-STARs, 2014, pp. $125-139$

[25] A. Weiler, M. Grossniklaus, M.H. Scholl, Event identification and tracking in social media streaming data, in: Proceedings of the EDBT Workshop on Multimodal Social Data Management (MSDM), 2014, pp. 282-287.

[26] A. Weiler, M. Grossniklaus, M.H. Scholl, SiCi explorer: situation monitoring of cities in social media streaming data, In: Proceedings of the EDBT Workshop on Mining Urban Data (MUD), 2014, pp. 369-370. 
[27] A. Weiler, M. Grossniklaus, M.H. Scholl, Evaluation measures for event detection techniques on twitter data streams, in: Proceedings of the British International Conference on Databases (BICOD), 2015 pp. 108-119.

[28] A. Weiler, M. Grossniklaus, M.H. Scholl, Run-time and task-based performance of event detection techniques for twitter, In: Proceedings of the International Conference on Advanced Information Systems Engineering (CAiSE), 2015, pp. 35-49.
[29] J. Weng B.-S. Lee, Event detection in twitter in: Proceedings of the International Conference on Weblogs and Social Media (ICWSM), 2011, pp. 401-408.

[30] C. Xia, R. Schwartz, K. Xie, A. Krebs, A. Langdon, J. Ting, M. Naaman, CityBeat: real-time social media visualization of hyper-local city data, In: Proceedings of the International Conference on World Wide Web (WWW) (Companion Volume), 2014, pp. 167-170. 